\title{
Discovery and Protein Modeling Studies of Novel Compound Mutations Causing Resistance to Multiple Tyrosine Kinase Inhibitors in Chronic Myeloid Leukemia
}

\author{
Zafar Iqbal ${ }^{1 *}$, Muhammad Absar ${ }^{1}$, Amer Mahmood ${ }^{2}$, Aamer Aleem ${ }^{3}$, Mudassar \\ Iqbal $^{4}$, Abid Jameel ${ }^{5}$, Tanveer Akhtar ${ }^{1}$, Sajjad Karim ${ }^{6}$, Mahmood Rasool ${ }^{6}$, Zeenat \\ Mirza $^{6}$, Muhammad Khalid ${ }^{7}$, Afia Muhammad Akram ${ }^{8}$, Muhammad Farooq \\ Sabar', Ahmad M Khalid ${ }^{10}$, Khalid Aljarrah ${ }^{11,12}$, Janhangir Iqbal ${ }^{13}$, Muhammad \\ Khalid $^{14}$, Ijaz H Shah ${ }^{14}$, Nawaf Alanazi ${ }^{11}$
}

\begin{abstract}
Objective: BCR-ABL fusion oncogene is the hallmark of chronic myeloid leukemia (CML), causing genomic instability which leads to accumulation of mutations in $B C R-A B L$ as well as other genes. $B C R-A B L$ mutations are the cause of tyrosine kinase inhibitors (TKIs) resistance in CML. Recently, compound BCR-ABL mutations have been reported to resist all FDA approved TKIs. Therefore, finding novel compound $B C R-A B L$ mutations can help and clinically manage CML. Therefore, our objective was to find out novel drug-resistant compound BCR-ABL mutations in CML and carry out their protein modelling studies. Methodology: Peripheral blood samples were collected from ten imatinib resistant CML patients receiving nilotinib treatment. BCR-ABL transcript mutations were investigated by employing capillary sequencing. Patient follow-up was carried out using European LeukemiaNet guidelines. Protein modeling studies were carried out for new compound mutations using PyMol to see the effects of mutations at structural level. Results: A novel compound mutation (K245N mutation along with G250W mutation) and previously known T351I utation was detected in two of the nilotinib resistance CML patients respectively while in the rest of 8 nilotinib responders, no resistant mutations were detected. Protein modelling studies indicated changes in BCR-ABL mutant protein which may have negatively impacted its binding with nilotinib leading to drug resistance. Conclusion: We report a novel nilotinib resistant BCR-ABL compound mutation (K245N along with G250W mutation) which impacts structural modification in BCR-ABL mutant protein leading to drug resistance. As compound mutations pose a new threat by causing resistance to all FDA approved tyrosine kinase inhibitors in BCR-ABL+ leukemias, our study opens a new direction for in vitro characterization of novel BCR-ABL compound mutations and their resistant to second generation and third generation TKIs.
\end{abstract}

Keywords: Nilotinib- BCR-ABL mutation- chronic myeloid leukemia- tyrosine kinase inhibitors

Asian Pac J Cancer Prev, 21 (12), 3517-3526

Introduction

Chronic myeloid leukemia, a myeloproliferative disorder, is reported in roughly $15 \%$ of newly diagnosed

\footnotetext{
${ }^{1}$ Hematology Oncology and Pharmacogenetics Engineering Sciences (HOPES) Group, Health Sciences Research Laboratories, Department of Zoology, University of the Punjab, Lahore, \& University of Education, Lahore, Pakistan. ${ }^{2}$ Department of Anatomy, College of Medicine and King Khalid University Hospital, King Saud University, Riyadh, Saudi Arabia. ${ }^{3}$ Hematology/Oncology Division, Department of Medicine, College of Medicine and King Khalid University Hospital, King Saud University, Riyadh, Saudi Arabia. ${ }^{4}$ Foreign Faculty, Asian Medical Institute, Kant City, National Surgical Centre, Bishkek, Kyrgyzstan, and Higher Education Commission Program in "Hematology Oncology and Pharmacogenetics Engineering Sciences (HOPES)", Kyrgyzstan. ${ }^{5}$ Post-Graduate Medical Institute, Hayatabad Medical Complex, Peshawar, Pakistan. ${ }^{6}$ Center of Excellence in Genomic Medicine Research \& Department of Medical Laboratory Technology, Faculty of Applied Medical Sciences, King Abdulaziz University, Jeddah, Saudi Arabia. ${ }^{7}$ Blood Bank, Peshawar, Pakistan. ${ }^{8}$ Department of Zoology, Division of Science and Technology, University of Education, Township, Lahore, Pakistan. ${ }^{9}$ Centre for Advanced Molecular Biology, University of the Punjab, Lahore, Pakistan.

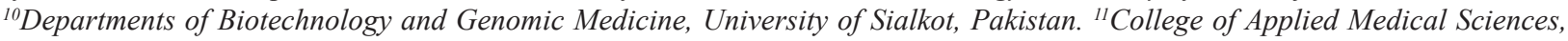
King Saud Bin Abdulaziz University for Health Sciences (KSAU-HS)/KAIMRC/SSBMT, National Guards Health Affairs, Al-Ahsa, Kingdom of Saudi Arabia. ${ }^{12}$ Jordan University of Science and Technology, Irbid, Jordan. ${ }^{13}$ National Guard Health Affairs, King Abdullah International Medical Research Centre (KAIMRC), Al-Ahsa, Saudi Arabia. ${ }^{14}$ Allied Hospital, Punjab Medical College \& Sahil Hospital, Faisalabad, Pakistan.*For Correspondence: drzafar.medgen@yahoo.com
} 
leukemia in adults (Izzo et al., 2019). BCR-ABL, an oncoprotein central to the pathogenesis of $\mathrm{CML}$, is fusion product of Abelson murine leukemia (ABL) and breakpoint cluster region $(B C R)$ genes located on chromosome 9 and 22 respectively (Rowley, 1973). BCR-ABL, a tyrosine kinase, is constitutively expressed to promote growth and replication (Himburg et al., 2019). Genomic instability in CML is well established that is primarily caused by BCR-ABL itself, and it leads to acquisition of further mutations in BCR-ABL itself as well as other genes with the clinical course of the disease (Slupianek et al., 2011; Wu et al., 2018).

Imatinib mesylate (Gleevec ${ }^{\circledR}$ USA; Glivec-Europe/ Australia; formerly STI571) is a BCR-ABL TKI which has intensely improved the treatment outcomes in chronic-phase CML patients (CML-CP). The drug inhibits BCR-ABL by binding to an inactive conformation of the kinase, preventing signal transduction and cellular proliferation (Druker et al., 2001; Druker et al., 1996). Regardless of the efficacy of imatinib in patients with $\mathrm{Ph}$-positive $(\mathrm{Ph}+) \mathrm{CML}-\mathrm{CP}$, approximately $15 \%$ of patients display resistance to imatinib or relapse after an initial therapeutic response and thus have less favorable long-term outcomes (Hochhaus et al., 2009). A simple mechanism of resistance to TKI treatment is owing to mutations in the kinase domain of BCR-ABL. Such mutations have been reported in $40 \%$ to $60 \%$ of imatinibresistant patients (Jabbour et al., 2006). More than 90 distinct mutations in BCR-ABL have been recognized, each conferring variable degrees of resistance to imatinib treatment (Soverini et al., 2010).

Nilotinib, a small rationally designed TKI by Tasigna; Novartis Pharmaceuticals Corporation, is good inhibitor of BCR-ABL and is approved for imatinib-resistant CML. in vitro study shown wide inhibitory activity of nilotinib against most of the imatinib-resistant mutants of BCR-ABL (Weisberg et al., 2006). Previous data have demonstrated that imatinib-resistant patients with CML-CP or CML-accelerated phase (AP) treated with nilotinib had high and durable rates of response for all mutations except T315I, Y253H, E255K/V, and F359C/V (Hughes et al., 2009; Kantarjian et al., 2011). In last few years, compound BCR-ABL mutations (multiple BCR-ABL mutations in a single molecule) have posed a new threat by causing resistance to all FDA approved TKIs including T315Imutation specific drug ponatinib (Kang et al., 2018). Studies related to compound BCR-ABL mutations can help in early determination of drug resistance, to find out complex mechanisms of multi-drug resistance and help in designing new drugs for multiple / compound mutant CML cases (Hochhaus et al., 2020; Soverini et al., 2020). Here we report a novel compound BCR-ABL mutation causing multi-TKI resistance CML as well as structural BCR-ABL changes induced by this mutant associated with multiple kinase inhibitor resistance.

\section{Materials and Methods}

Patients and blood sample collection

A total of $10 \mathrm{CML}$ imatinib resistant patients who had received nilotinib treatment for at least 12 months or more, from Allied Hospital Faisalabad, Pakistan, were included in this study. Number of patients was low as very few patients could afford nilotinib, a very expensive drug. Three ml blood was taken in labeled EDTA tubes per manufacturer's instructions. Blood was processed within 24 hours to ensure minimum degradation of mRNA, otherwise stored at $-40^{\circ} \mathrm{C}$. Randomization was stratified according to the Sokal risk score at the time of diagnosis. This study was approved by the local ethics committee or review board and was conducted in accordance with the Declaration of Helsinki with written consent form each patient.

\section{Clinical history and patient's data}

A complete clinical history since the diagnosis of CML were collected in patient data forms and required permission and consent were taken from hospital authorities and participating patient. Patients were routinely followed-up to record hematologic and cytogenetic response on monthly basis. We followed European Leukemia Net guidelines for categorizing CML patient samples into drug sensitive or resistant on the basis of their response to therapy (Baccarani et al., 2014).

\section{RNA extraction from blood samples}

RNA was extracted from patient blood using protocols described previously (Chomczynski et al., 1987; Liedtke et al., 1994). All RNA extraction steps were carried out on crushed ice to evade degradation of nucleic acid. Isolated RNA was stored immediately at $-40^{\circ} \mathrm{C}$ till further processing.

\section{Qualitative analysis of $R N A$}

Quality of RNA was assessed by performing native agarose gel electrophoresis (Biocom and PowerPac Basic, BIO-RAD) and RNA was observed as two discrete fluorescent bands (28S and 18S) (UVP PhotoDoc-ItTM Imaging System).

\section{Complementary DNA synthesis}

cDNA was synthesized by reverse transcription of RNA previously described protocol with slight variations (Thermo scientific ${ }^{\mathrm{TM}}$, USA) (van Dongen et al., 1999).

\section{cDNA integrity check}

Integrity of cDNA was assessed by amplification of housekeeping gene, glyceraldehyde 3-phosphate dehydrogenase $(G A P D H)$ using forward: 5'-ACCACAGTCCATGCCATCA-3' and reverse: 5'-TCCACCACCCTGTTGCTGTA-3' primers (Thermo Scientific ${ }^{\mathrm{TM}}$, USA and Applied Biosystems 2720 thermal cycler) (Asad et al., 2012).

\section{Nested reverse transcription PCR (nRT-PCR)}

Amplification of BCR-ABL fusion oncogene was performed by nRT-PCR (Applied Biosystems 2720 thermal cycler) using long range enzyme (Thermo ScientificTM, USA). For comparing CML patients $(\mathrm{n}=10)$ with control, we amplified the kinase domain of $A B L$ gene from healthy population $(\mathrm{n}=20)$. Primer sequences and PCR reaction conditions were followed from Willis et al., 
2005 (Willis et al., 2005), with slight variations (Table 1).

Purification (Gel extraction) of amplified PCR products

To avoid any contamination of non-specific DNA fragments during sequencing, PCR products were purified from agarose gel (Quick gel extraction Kit, Invitrogen) and visualized (UVP PhotoDoc-ItTM Imaging System). Needed PCR products of specific size were carefully excised, and purified PCR products were stored at $-20^{\circ} \mathrm{C}$ for sequencing or further use.

\section{Direct Sequencing analysis of PCR amplicons}

To detect any likely point mutation in ABL-kinase domain of CML patients, we directly sequenced the amplified products by Sanger's method using 5' - TGGT TCATCATCAT TCAACGG-3' and 5'-GGACATGCCATAGGTAGCA-3' primer pair (ABI-3130XL and ABI-3730 genetic analyzers, Applied Biosystems, USA) (Willis et al., 2005).

\section{Analysis of sequencing data}

To find out any polymorphic variations in our population, the ABL kinase domain DNA sequences of healthy individuals were aligned against the reference sequence (NCBI's GenBank Accession Number: M14752.1). Polymorphic variations were excluded while detecting point mutations in CML patient's sequences (Geneious R7). Entire kinase domain sequence including P-loop, C-Helix, SH2 contact and activation loop was explored to identify any probable nucleotide substitutions responsible for altered response to imatinib treatment.

\section{Statistical analysis}

Fisher's exact test (Chi-square test) was done for comparing patient's response (sensitivity or resistance) towards therapy and mutation status among different clinical categories or predictors of CML patients (IBM SPSS version 19, Chicago, IL, USA.).

\section{Bioinformatics Analysis}

Structural bioinformatics analysis was done for the BCR-ABL protein for structurally validating our findings. Crystallographically determined human ABL

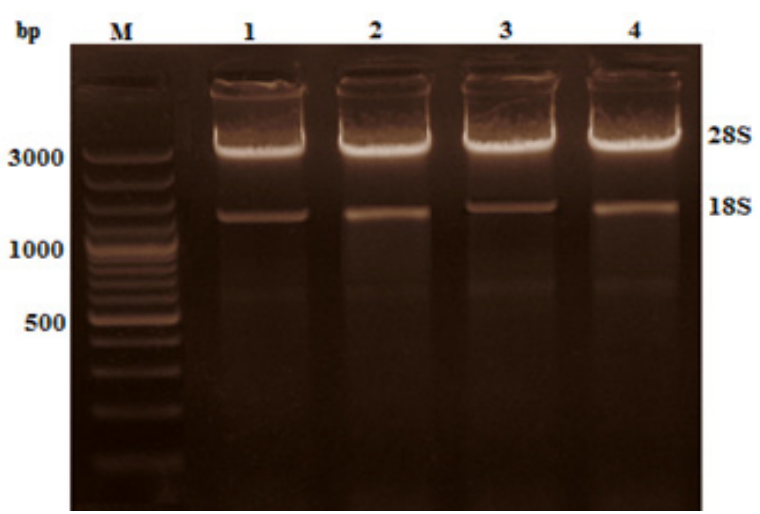

Figure 1. Visualization of Total RNA Isolated from Blood Samples of CML Patients on 1.5\% Agarose Gel. Intact 28S and 18S RNA were observed. bp= Base pairs, $\mathrm{M}=$ DNA marker. kinase protein structure in complex with nilotinib was retrieved from worldwide archive of structural data of biological macromolecules, RCSB's PDB (PDB ID: 3cs9) and viewed using Delano Scientific's PyMOL (DeLano, 2002). Further modeling studies were done to find out structural changes responsible for drug resistance. Modeling studies for structure and function prediction of the mutant structure was done using I-Tasser server with alignment generated using MUSTER threading program (Laskowski and Swindells, 2011). Detailed structural visualization and comparative alignment was done for the wild type and mutant modeled using PyMOL. LigPlot+ v.1.4.5 program (EBI, Cambridge, UK) was used to check and schematically plot the polar and hydrophobic interactions between the protein BCR-ABL kinase and the inhibitor nilotinib (Cortes et al., 2012). The 3-D structure of the protein-ligand complex was given as an input in PDB format and the software displayed their interacting residues and bonds.

\section{Results}

Patient demographics, clinical and lab oratory findings

Peripheral-blood samples were collected from imatinib resistant CML patients aged $\geq 18$ years who were receiving nilotinib (400 mg twice daily) for at least 12 months. Clinical characteristics of CML patients, wherein all were in chronic phase CML have been summarized in Table 2. The response to nilotinib was determined by examining the clinical end points: complete hematological response (CHR), and complete cytogenetic response (CCyR, $n=2)$. Number of male patients was more than females with male: female ratio of $8: 2$. Splenomegaly $(80 \%)$ was the most common symptom followed by fever, weight loss (40\%), fatigue and hepatomegaly $(20 \%)$. The mean age of patients in the present study was 37.2 years (age range: 28-50 years). White cell count ranging within normal values $\left(0.5-1.0 \times 10^{9} / \mathrm{L}\right)$ was observed in 2 patient, while 8 individuals had more. Eight patients had platelet count between $100-450 \times 10^{9} / \mathrm{L}$, while 2 had more than $450 \times$ $109 /$ L. Six patients had blast cells less than $5 \%$ and four patients had their hemoglobin level $<10 \mathrm{~g} / \mathrm{dl}$ ). Sokal risk scores were low of six, intermediate for two and high

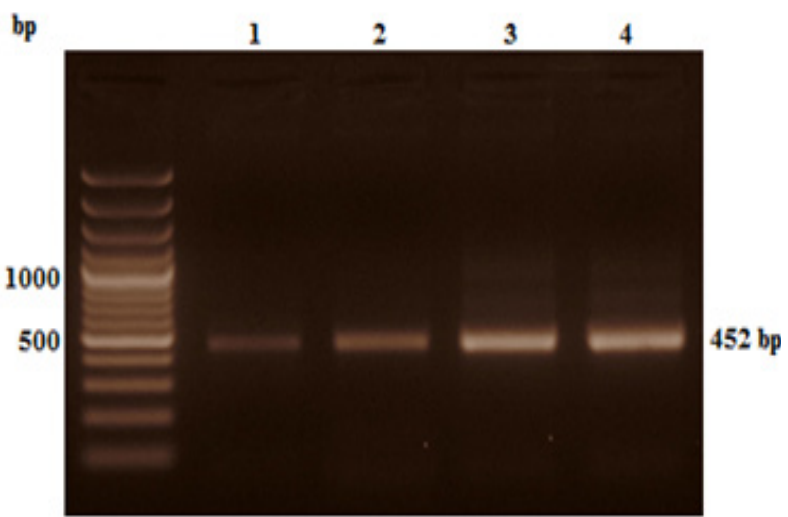

Figure 2. Visualization of GAPDH Amplification to Check cDNA Quality and Integrity on $1.5 \%$ Agarose Gel; $b p=$ Base pairs, $M=$ DNA marker 
(b)

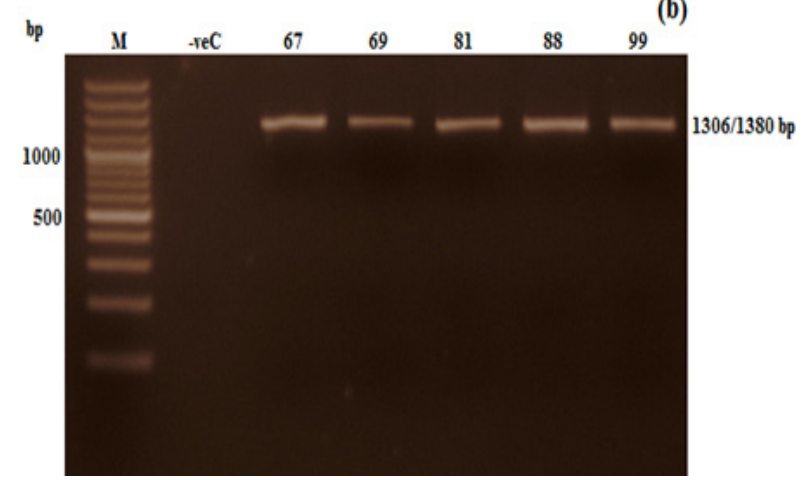

Figure 3. Visualization of Nested PCR (round 2) Products of Nilotinib Sensitive Wild Type (a) and nilotinib sensitive mutant (b) CML patients on $1 \%$ agarose gel. $b p=$ Base pairs, $\mathrm{M}=$ DNA marker, $-\mathrm{veC}=$ Negative control.

for two CML patients. Hematological response was complete for eight and not for two, while cytogenetic response was four, four and two for complete, partial and minor respectively. No statistically significant association $(p=<0.05)$ was observed in the different parameters of the studied subjects.

RNA extraction and Nested RT-PCR amplification of $B C R$-ABL kinase domain

The total RNA isolated from blood samples of CML patients was run on $1.5 \%$ agarose gel to check the integrity of extracted RNA (Figure 1). The PCR quality of cDNA was evaluated by running the amplification
Table 1. Primer Sequences for Amplification of BCR/ ABL-kinase Domain by Nested RT-PCR.

\begin{tabular}{lcc}
\hline $\begin{array}{l}\text { Nested } \\
\text { PCR }\end{array}$ & Primer name & Primer Sequence $\left(5^{\prime}-3^{\prime}\right)$ \\
\hline $\begin{array}{l}\text { Round } \\
1\end{array}$ & B2A forward & 5'-TTCAGAAGCTTCTCCCTGACAT-3' \\
& $\begin{array}{c}\text { ABL4065 } \\
\text { reverse }\end{array}$ & 5'-CCTTCTCTAGCAGCTCATACACCTG -3' \\
Round & BCR F4 & 5'-ACAGCATTCCGCTGACCATCAATA-3' \\
2 & forward & \\
& U396 reverse & 5'-GCCATAGGTAGCAATTTCCC-3' \\
\hline
\end{tabular}

product of GAPDH housekeeping gene on $1.5 \%$ agarose gel (Figure 2). The nested PCR of the $B C R-A B \mathrm{~L}$ gene was successfully amplified in all samples at the end of second round nested RT-PCR. Nested PCR products of nilotinib sensitive wild type (a) nilotinib sensitive mutant (b) CML patients were run on $1 \%$ agarose gel. As CML patients had two common variants of fusion oncogene, the resulting products in different samples were $1306 \mathrm{bp}$ (b2a2) and $1380 \mathrm{bp}$ (b3a2) in size (Figure 3).

\section{Direct DNA Sequencing}

Direct sequencing analysis of the amplified PCR products was performed to detect point mutations in the ABL-kinase domain of CML cases. The electropherogram showing compound mutations, including a novel BCR-ABL mutation associated with primary nilotinib resistance in CML patient (Figure 4). The nucleotide changes and their subsequent amino acid substitutions are shown by blue longitudinal indicator in each sample along with the nucleotide number. NCBI GenBank accession

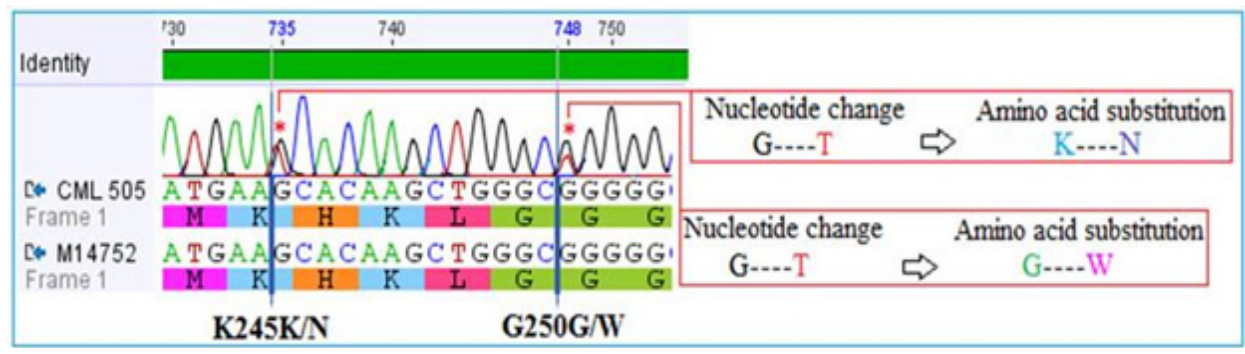

Figure 4. Electropherogram Showing Compound Mutations, Including a Novel BCR-ABL Mutation Associated with Primary Nilotinib Resistance in CML Patient.

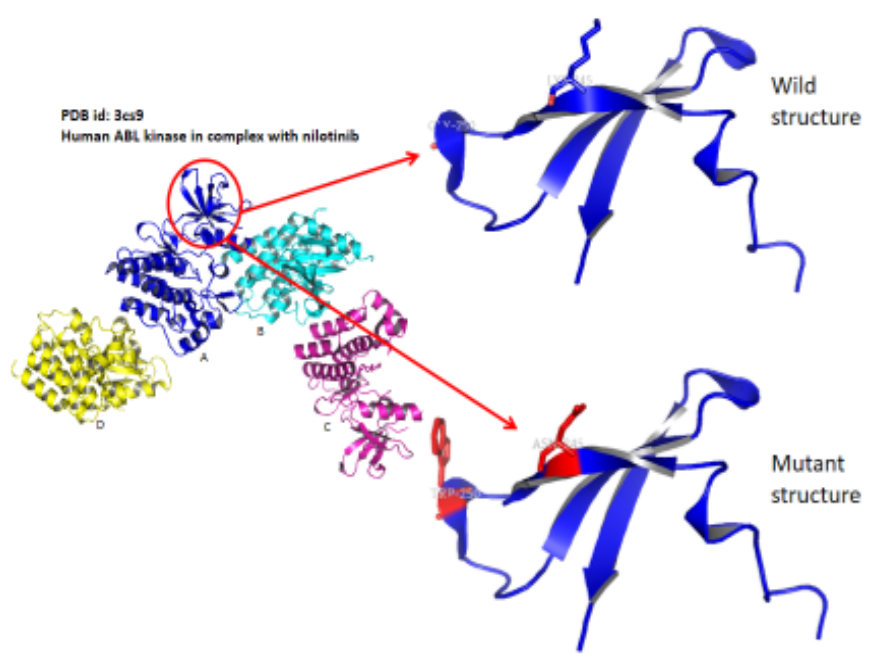

Figure 5. Mutant Structure was Made Using PyMol 
Table 2. Clinical Characteristics of CML Patients at Time of Disease Diagnosis and Patients Response (Hematologic and Cytogenetic) at the Time of Blood Sample Collection

\begin{tabular}{|c|c|c|c|}
\hline \multicolumn{4}{|l|}{ Patient Characteristics N (\%) } \\
\hline \multirow[t]{18}{*}{ Clinical and laboratory characteristics at diagnosis } & \multirow[t]{4}{*}{ Gender } & Male & $8(80)$ \\
\hline & & Female & $2(20)$ \\
\hline & & Spleen enlargement & $8(80)$ \\
\hline & & Fever & $4(40)$ \\
\hline & \multirow[t]{3}{*}{ Symptoms } & Hepatomegaly & $2(20)$ \\
\hline & & Fatigue & $2(20)$ \\
\hline & & Weight loss & $4(40)$ \\
\hline & \multirow[t]{2}{*}{ White cell count $\left(\mathrm{x} 10^{9} / \mathrm{L}\right)$} & 10-May & $2(20)$ \\
\hline & & $>10$ & $8(80)$ \\
\hline & \multirow[t]{2}{*}{ Platelet count $($ x 109/L) } & $100-450$ & $8(80)$ \\
\hline & & $>450$ & $2(20)$ \\
\hline & \multirow[t]{2}{*}{ Blast cells in peripheral blood } & Less than $5 \%$ & $6(60)$ \\
\hline & & More than $5 \%$ & $4(40)$ \\
\hline & \multirow[t]{2}{*}{ Hemoglobin in peripheral blood } & $<10 \mathrm{~g} / \mathrm{dl}$ & $4(40)$ \\
\hline & & $>10 \mathrm{~g} / \mathrm{dl}$ & $6(60)$ \\
\hline & \multirow[t]{3}{*}{ Sokal risk score } & Low & $6(60)$ \\
\hline & & Intermediate & $2(20)$ \\
\hline & & High & $2(20)$ \\
\hline \multirow{8}{*}{$\begin{array}{l}\text { Patient's response at the time of blood sample } \\
\text { collection }\end{array}$} & \multirow[t]{3}{*}{ Phase at the time of blood collection } & Chronic & $10(100)$ \\
\hline & & Accelerated & 0 \\
\hline & & Blast & 0 \\
\hline & \multirow[t]{2}{*}{ Hematological Response (HR) } & Complete & $8(80)$ \\
\hline & & No & $2(20)$ \\
\hline & \multirow[t]{3}{*}{ Cytogenetic Response (CyR) } & Complete & $4(40)$ \\
\hline & & Partial & $4(40)$ \\
\hline & & Minor & $2(20)$ \\
\hline
\end{tabular}

number: M14752.1 is the reference sequence.

Molecular Structural Studies: Molecular modeling were done to find out structural changes induced in wild type (Lys245, Gly250) mutated to mutant type (Asp245, Trp250) responsible for conferring drug resistance. Our

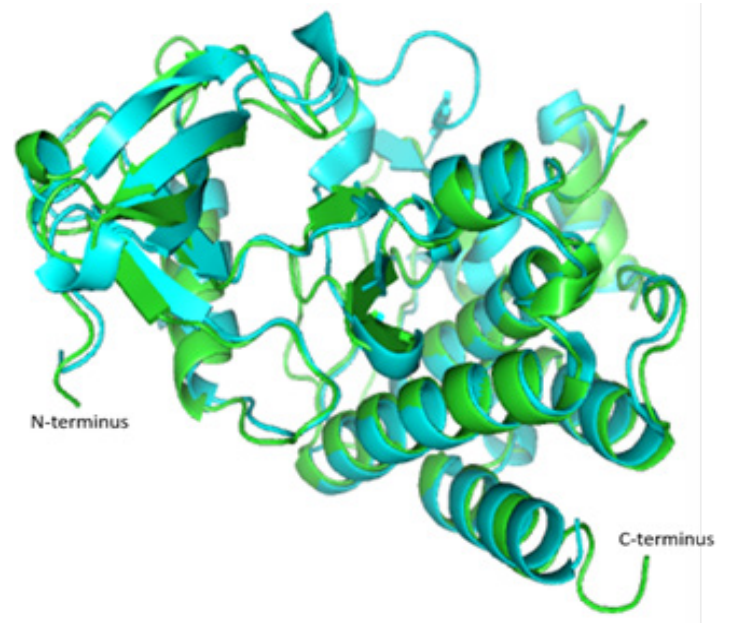

Figure 6. Structural Alignment Done Using PyMol of Wild (PDB id: 3cs9) and Mutant (Modeled) Protein. In the overlapped structure, green represents mutant and cyan represents the wild type. residues of interest lies within the glycine-rich phosphate loop (P-loop) traversing from 242-256 constitute (Figure 5,6 , and 7). Ligplot analysis was carried to check the schematic 2-D molecular interactions between kinase protein and the bound ligand nilotinib (Figure 8). The primarily interacting residues were Glu286, Thr315, Met318 and Asp381.

\section{Discussion}

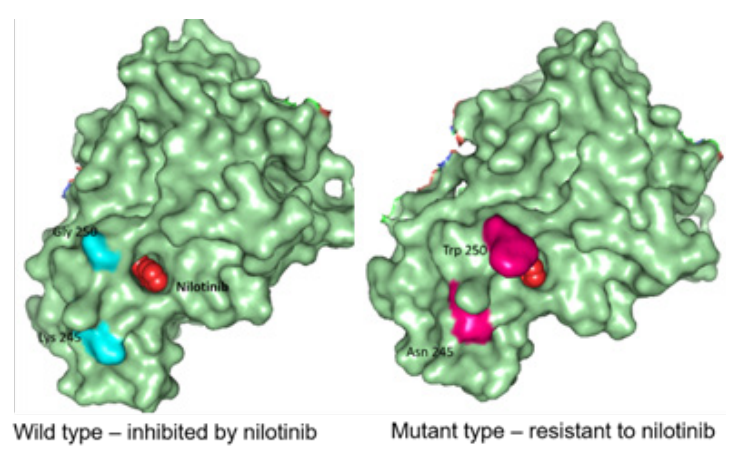

Figure 7. Comparative Structure Analysis Highlighting the Hydrophobic Pocket with the Mutation Induced Topographical Difference in the Two Cases 


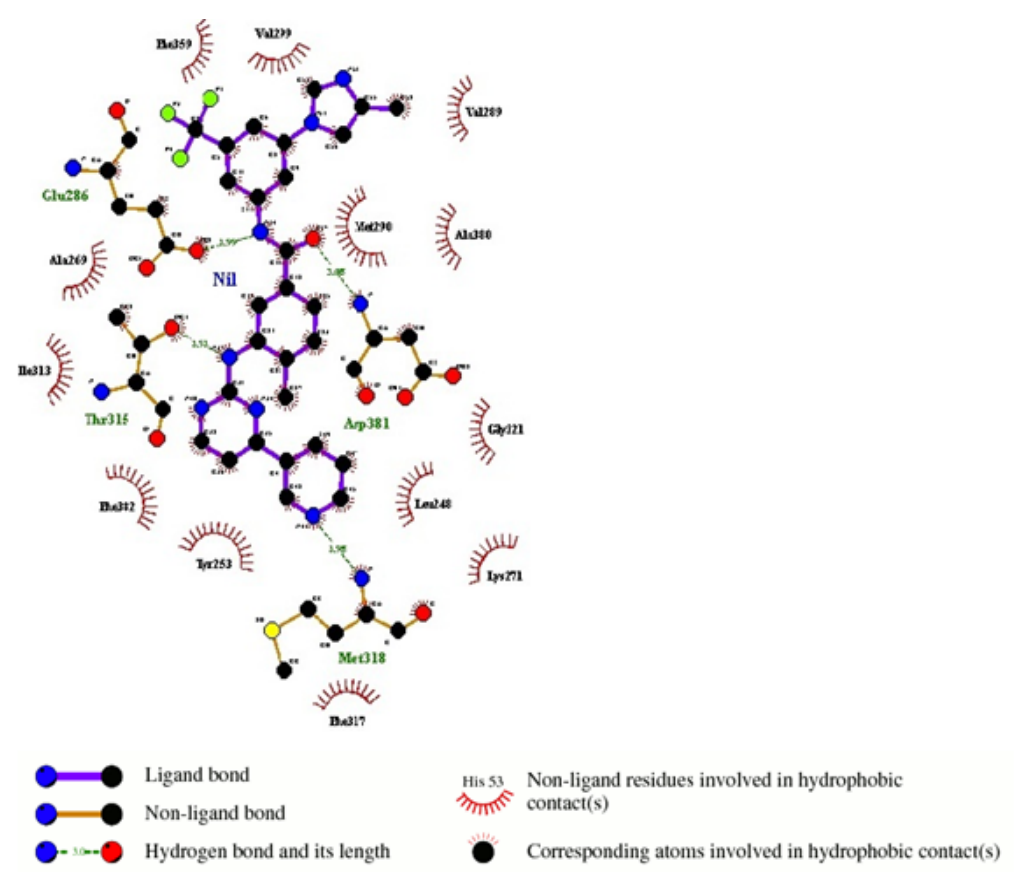

Figure 8. Schematic 2D Binding Mode Representation with the Ligand Nilotinib as Generated by Ligplot+ v.1.4.5

In the current study all CP-CML patients who were receiving nilotinib ( $\geq 12$ months) second line TKI therapy after imatinib resistance were included and investigated for any mutations related to nilotinib resistance. Male to female ratio was $4: 1$, respectively. In previous literature incidence of CML with male-to-female ratio of 1.1:1 to 1.74:1 had been reported (Aziz et al., 2010; Jacobs et al., 2018). The altered ratio in present study is owing to small number of available patients on nilotinib therapy. Enlargement of spleen was the most commonly observed symptom in the patients during clinical examination $(80 \%)$. This finding is in concordance with the conclusions that massive splenomegaly is often associated with CML (Efficace et al., 2011). The incidence of other signs and symptoms; including fever, hepatomegaly and fatigue etc. varies in literature and is stated to be $40-64.4 \%, 21.7-100 \%$ and $22-100 \%$, respectively (Aguayo et al., 2008; Karimi et al., 2008).

Among the patients included in the present studies, $80 \%$ showed a massive over-production of WBCs ranging between $12-670 \times 10^{9} / \mathrm{L}$ at the time of diagnosis. In literature, leukocytosis $\left(>10 \times 10^{9} / \mathrm{L}\right)$ has been stated between $40.7--77.3 \%$ of patients with CML $[29,35]$. The platelet counts ranged between 105-694 x 10\%/L. Only one patient had platelet count $>450 \times 10^{9} / \mathrm{L}$ i.e. $694 \mathrm{x}$ $10^{9} / \mathrm{L}(20 \%)$ which is in agreement to the reported range of platelet count in CML patents (10.2-21.4\%) (Jabbour et al., 2011; Jacobs et al., 2018). Hemoglobin level lower $10 \mathrm{~g} / \mathrm{dl}$ was evident in 2 patients (40\%) In cancer patient's low $\mathrm{Hb}$ level has been reported in range of $15-59.3 \%$ of CML patients (Jacobs et al., 2018; Jabbour et al., 2017).

The monitoring of cytogenetic response (CyR) in CML has a prognostic value and CCyR achievement has been linked with prolonged survival (Druker et al., 2006). In the present study, patients exhibiting CCyR, partial CyR and minor CyR was $40 \%, 40 \%$ and $20 \%$, respectively.
Similarly, HR is also important in CML response monitoring. In the present study, CP-CML patients showed CHR to be $80 \%$. In literature, HR has been reported to be 90\% 53 and 93.75\% (Gupta and Prasad, 2007; Hochhaus and Kantarjian, 2013).

In the present study, 1 out $5 \mathrm{CP}-\mathrm{CML}$ patients treated with nilotinib $400 \mathrm{mg}$ twice daily, compound mutations, including a novel $K 245 \mathrm{~N}$ mutation and $G 250 \mathrm{~W}$ mutation in BCR-ABL domain were detected. The presence of this compound mutations most likely have contributed to nilotinib resistance in this patient. This is the first report of such novel mutation occurring in $B C R-A B L$. The nilotinib resistant patient progressed to accelerated phase (AP), however, mutations alone are not a cause of disease progress to advanced stage or treatment failure and alternative mechanisms may be involved (Melo and Barnes, 2007). Furthermore, we cannot confirm whether treatment-emergent mutation in our case is solely a driver mutation and cause of disease progress to advance stage. The remaining patients $(n=4)$ of nilotinib responders did not show any mutations. G250E (P-loop) mutation has been reported in CML patients treated with either imatinib or nilotinib (Melo and Barnes, 2007).

Although BCR-ABL inhibitor based treatment helps majority of CML patients to lead normal life till treatment continue but unfortunately few patients i.e. $20-30 \%$ develop TKI resistance, either primary or secondary and are prone to progress toward the AP or BP of the disease (Kang et al., 2006). BCR-ABL mutations are the major background players in manifestation of resistance to all FDA approved TKIs including imatinib, dasatinib and nilotinib (Hochhaus et al., 2020; Baccarani et al., 2014). According to European or North American clinical guidelines, detection of mutations is a vital during drug resistance or drug switching, because nature of mutation determine the resistance or sensitivity for different drugs. So in case of resistance or unsatisfactory response to 
$B C R-A B L$ inhibitors, mutational information can help in deciding better alternative TKI. Till date four main mutational regions for TKIs resistance have been reported: P-loop, SH3 contact (3) SH2 contact and (4) A-loop (Kang et al., 2006; Druker etal., 2001).

Nilotinib, a second-generation inhibitor, has greater potency and specificity (approximately 20 fold) for wild type BCR-ABL than imatinib (Blay et al., 2011). It binds to ABL tyrosine kinase and disrupt the ATP-phosphatebinding site causing inhibition of catalytic activity of enzyme. Other than T315I mutation, it inhibits all imatinib-resistant mutants (Hamad et al., 2013). Clinically, nilotinib based treatment is recommended by ELN for all newly diagnosed $\mathrm{Ph}+\mathrm{CML}-\mathrm{CP}$ and imatinib intolerant $\mathrm{Ph}+\mathrm{CML}$ in $\mathrm{CP}$ or AP patients. Better understanding of reasons and mechanism of resistance will improve the treatment strategy(Hamad et al., 2013). Nilotinib is good suppressor of overall resistance causing $B C R-A B L$ mutations (Marchesi et al., 2013), and found to sensitive against $\mathrm{D} 816 \mathrm{~V}$ mutant variant, which is resistant to imatinib (Weisberg et al., 2007). Blast crisis stage of patients need more attention as about $40-90 \%$ of $B C R$ $A B L$ mutation in imatinib resistant patients detected here (Soverini et al., 2020).

Coexistence of two or more mutations in the same $B C R-A B L m R N A$, is termed as a "compound mutation". Patients harboring compound mutations like V299L, T315A, F317L/V/I/C17 (Soverini et al., 2006; Burgess et al., 2005) were found to be less sensitive to dasatinib therapy and nilotinib treatment failure has been reported in several studies in patients with compound mutations like $E 255 \mathrm{~K} / \mathrm{V}, \mathrm{Y253H}$, F359V/C/I [Hughes et al., 2009; Soverini et al., 2009; Khorashad et al., 2013).

Khorashad et al., (2013) analyzed BCR-ABL kinase domain mutations in 1,700 samples by direct DNA sequencing and found that $11.4 \% \mathrm{CML}$ patient samples harbored $\geq 2$ mutations. In total, 30 different compound mutations were observed, with the most frequent mutation being T315I among all compound mutants. These mutations existed in two forms: compound or polyclonal. Compound mutations (multiple mutations in the same $B C R-A B L$ molecule) accounted for $70.2 \%$ and $29.8 \%$ were polyclonal mutations (mutations occurring in multiple BCR-ABL molecules). Therefore, the distinction between compound versus single mutations is clinically important because it may influence the selection of the most suitable TKI to overcome resistance. Several compound mutations have been shown to confer resistance to ponatinib and this is likely to apply to other third-line TKIs as well (O'Hare et al., 2009; Yang et al., 2014).

Yang et al., (2014), evaluated the effect of nilotinib on two CML imatinib resistant patients with V299L mutation. Nilotinib treatment successfully turned $V 299 L$ mutation of both cases into negative. Acquired $B C R-A B L$ mutations related to nilotinib treatment in patients with CML was initially available from two meeting abstracts (Hughes et al., 2007). The first of these described $F 349 \mathrm{~V}, \mathrm{E} 255 \mathrm{~K} / \mathrm{V}$, E355G, G250E, M244V and T315I. The second report described $Y 253 F$, E255V, T315I, and F359V as emerging mutations. Many mutations developing in response to nilotinib have now been described in literature allowing improved interpretation. These mutations include: T315I, Y253C, Y253H, E255V, L248V, K285N, E282K, E255K, F359C, F359V, K247N, W430L, T406I, E255R, A380S, Y253F, Q252H, L387F, F311V, L273F, H375P, H396P, E292K, D276G, E275K, L387M, G250E, E431G, V379I, Q252H, F317L, F311L, N297T, E355A, F317V, H396R, G250A, E355G, F486S, V289L, M244V, D325N, M388L, F317C, G250V, M237I, S348L, E255D, E281K, M351T, E453K, E344G, E459Q, F311I, A433T, F349V, E292V, L384M, E274K, L370P, M388I, E450K, S438C, E459G, $E 459 K$ (Khorashad et al., 2013; Hughes et al., 2007), and F359I/V, E279K, L248L/V (Ursan et al., 2015).

A systematic literature search was performed by Ursan et al., (2015) between January 1966 and May 2013, regarding use of nilotinib as first line TKI in CML patients. A single study of nilotinib was found in this search as firstline treatment in CML that included 61 patients, in whom the mutation incidence was 3.3\% (95\% CI $=0.0 \%-7.7 \%)$. In these patients (n=61), E255K and $F 359 C$ mutations were reported and experienced disease progression (Branford et al., 2009). Clinically relevant mutations to second-generation inhibitors were $F 317 \mathrm{~L} / \mathrm{I} / \mathrm{C} / \mathrm{V}, \mathrm{V} 299 \mathrm{~L}$,

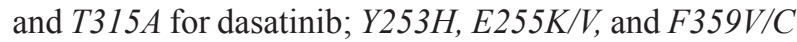
for nilotinib; and T315I for both TKIS (Goodrich, 2014).

A $3^{\text {rd }}$ generation molecule such as ponatinib, has been approved by FDA and can inhibit majority of mutations in kinase domain. However, it's unlikely that ponatinib will be used for treatment because of drug dosing issue (Yang et al., 2014). Additionally, ponatinib had been found to be vulnerable to couple of compound mutations like $G 250 E$ / T315I and E255V/T315I. These BCR-ABL1 compound mutants causing high-level resistance against ponatinib is next significant challenge to $2^{\text {nd }}$ and $3^{\text {rd }}$ generation TKIs in CML patients (Marchesi, 2013; Eide et al., 2011; Azam et al., 2009).

Protein structure modeling of Human ABL kinase - nilotinib complex was done to find out structural changes responsible for resistance. The residues spanning from 242-256 constitute the glycine-rich phosphate loop (P-loop) that facilitates the transphosphorylation reaction. Residues of present interest (245 and 250) are not directly involved in binding with the drug. However, may contribute to the development of resistance against nilotinib in the mutated protein. Substitution mutation (glycine, the smallest amino acid to the bulkiest aromatic tryptophan) will potentially hinder the smooth entry of the drug into the binding cavity. Additionally, the mutation in the surface residue from basic Lys to acidic Asn might also interfere with inhibitor binding. In past, BCR-ABL protein modelling studies have led to unravel mechanisms of TKI resistance in CML and to design novel drugs to treat drugresistant CML (Ozkan et al., 2018; Altıntop et al., 2017). We hope that reported compound $B C R-A B L$ mutations and its protein modelling studies will help unravel complex mechanism of drug resistance and to design more effective drugs for CML. Further studies on compound $B C R-A B L$ mutations, specifically resistance to $2^{\text {nd }}$ and $3^{\text {rd }}$ generation TKI resistance, by employing genomic techniques will lead to better clinical management of recent challenges in CML treatment (Hochhaus et al., 2020; Soverini et al., 2020).

Asian Pacific Journal of Cancer Prevention, Vol 21 
In summary, novel compound mutations resulted due to innate genomic instability in $B C R-A B L+C M L$ cells leading to nilotinib resistance have been reported in our studies. Protein modelling studies showed that mutations caused structural changes in BCR-ABL protein which may have impacted binding of drug to the BCR-ABL target and led to nilotinib resistance. It is important to perform mutational analysis prior to drug switching and distinction between compound versus single mutation because it may influence the clinical selection of the most suitable TKI to overcome resistance. Studies on novel compound $B C R-A B L$ mutations associated with multiple TKI resistance will help understand mechanism of drug resistance in CML in more depth and will lead to better drug resistance management strategies to improve CML survival, possibly leading to a potential cure

\section{Acknowledgements}

This work was partially supported by the College of Medicine Research Center, Deanship of Scientific Research, King Saud University, Riyadh, Saudi Arabia. This Work was funded by the National Plan for Science, Technology and Innovation (MAARIFAH), King AbdulAziz City for Science and Technology, Kingdom of Saudi Arabia, grant Number 14-Med2817-02.

\section{Conflict of interest}

Authors declare no conflict of interest.

\section{References}

Aguayo A, Garcia-Alvarez E, Cazares-Ordonez Y, et al (2008). Chronic myeloid leukemia: a clinicoepidemiologic and therapeutic description of a single institution in Mexico City. Clin Leuk, 2, 261-6.

Altıntop MD, Ciftci HI, Radwan MO, et al (2017). Design, Synthesis, and Biological Evaluation of Novel 1,3,4-Thiadiazole Derivatives as Potential Antitumor Agents against ChronicMyelogenous Leukemia: Striking Effect of Nitrothiazole Moiety. Molecules, 23, pii: E59. doi: 10.3390/ molecules23010059.

Asad S, Ijaz B, Ahmad W, et al (2012). Development of persistent HCV genotype 3 a infection cell culture model in huh-7 cell. Virol J, 9, 11.

Azam M, Powers JT, Einhorn W,et al (2010). AP24163 inhibits the gatekeeper mutant of BCR-ABL and suppresses in vitro resistance. Chem Biol Drug Des, 75, 223-7.

Aziz F, Qureshi IZ (2008). Clinical and cytogenetic analyses in Pakistani leukemia patients. Pakistan J Zool, 40.

Aziz Z, Iqbal J, Bano K, Faisal M, Akram M (2010). Sustained superior long-term outcomes and cytogenetic responses with imatinib mesylate in chronic phase chronic myeloid leukaemia: report from a developing country. Jpn J Clin Oncol, 40, 549-55.

Baccarani M, Soverini S, De Benedittis C (2014). Molecular monitoring and mutations in chronic myeloid leukemia: how to get the most out of your tyrosine kinase inhibitor. American Society of Clinical Oncology educational book American Society of Clinical Oncology Annual Meeting. pp 167-75.

Blay JY, von Mehren M (2011). Nilotinib: a novel, selective tyrosine kinase inhibitor. Semin Oncol, 38, 1:S3-9.

Branford S, Melo JV, Hughes TP (2009). Selecting optimal second-line tyrosine kinase inhibitor therapy for chronic myeloid leukemia patients after imatinib failure: does the BCR-ABL mutation status really matter?. Blood, 114, 5426-35.

Burgess MR, Skaggs BJ, Shah NP, Lee FY, Sawyers CL (2005). Comparative analysis of two clinically active BCR-ABL kinase inhibitors reveals the role of conformation-specific binding in resistance. Proc Natl Acad Sci U S A, 102, 3395400.

Chomczynski P, Sacchi N (1987). Single-step method of RNA isolation by acid guanidinium thiocyanate-phenolchloroform extraction. Analy Biochem, 162, 156-9.

Cortes J, Jabbour E, Kantarjian H, et al (2007). Dynamics of BCR-ABL kinase domain mutations in chronic myeloid leukemia after sequential treatment with multiple tyrosine kinase inhibitors. Blood, 110, 4005-11.

Cortes JE, Jones D, O'Brien S, et al (2010). Results of dasatinib therapy in patients with early chronic-phase chronic myeloid leukemia. J Clin Oncol, 28, 398-404.

Cortes JE, Kim DW, Kantarjian HM, et al (2012). Bosutinib versus imatinib in newly diagnosed chronic-phase chronic myeloid leukemia: results from the BELA trial. JClin Oncol, 30, 3486-92.

DeLano WL (2002). The PyMOL molecular graphics system. http://www pymol org. 2002.

Druker BJ, Guilhot F, O'Brien SG, et al (2006). Five-year follow-up of patients receiving imatinib for chronic myeloid leukemia. N Engl J Med, 355, 2408-17.

Druker BJ, Sawyers CL, Kantarjian H, et al (2001). Activity of a specific inhibitor of the BCR-ABL tyrosine kinase in the blast crisis of chronic myeloid leukemia and acute lymphoblastic leukemia with the Philadelphia chromosome. N Engl J Med, 344, 1038-42.

Druker BJ, Tamura S, Buchdunger E, et al (1996). Effects of a selective inhibitor of the Abl tyrosine kinase on the growth of Bcr-Abl positive cells. Nat Med, 2, 561-6.

Efficace F, Baccarani M, Breccia M, et al (2011). Health-related quality of life in chronic myeloid leukemia patients receiving long-term therapy with imatinib compared with the general population. Blood, 118, 4554-60.

Eide CA, Zabriskie MS, Adrian LT, et al (2011). Resistance profiling of BCR-ABL compound mutations linked to tyrosine kinase inhibitor therapy failure in chronic myeloid leukemia. Am Soc Hematol, 2011.

Golemovic M, Verstovsek S, Giles F, et al (2005). AMN107, a novel aminopyrimidine inhibitor of Bcr-Abl, has in vitro activity against imatinib-resistant chronic myeloid leukemia. Clin Cancer Res, 11, 4941-7.

Goodrich AD (2014). Ponatinib in the leukemia world: why a reevaluation is necessary for Philadelphia chromosomepositive patients with T315I mutation. Exp Rev Hematol, 7, 513-5.

Gupta A, Prasad K (2007). Hematological and molecular response evaluation of CML patients on imatinib. JAPI, 55, 109-13.

Gupta N, Gupta R, Sharawat SK, Bakhshi S (2010). Childhood chronic myeloid leukemia with monocytosis. Indian $J$ Pediat, 77, 1143-5.

Hamad A, Sahli Z, El Sabban M, Mouteirik M, Nasr R (2013). Emerging therapeutic strategies for targeting chronic myeloid leukemia stem cells. Stem Cells Int, 2013;2013:724360. doi:10.1155/2013/724360.

Himburg HA, Roos M, Fang T, et al (2009). Chronic myelogenous leukemia stem cells require cell-autonomous pleiotrophin signaling. J Clin Invest, Oct 15. pii: 129061. doi: 10.1172/JCI129061.

Hochhaus A, Breccia M, Saglio G, et al (2020). Expert opinion- 
management of chronic myeloid leukemia after resistance to second-generation tyrosine kinase inhibitors. Leukemia, doi: 10.1038/s41375-020-0842-9. Epub ahead of print. PMID: 32366938.

Hochhaus A, Kantarjian H (2013). The development of dasatinib as a treatment for chronic myeloid leukemia (CML): from initial studies to application in newly diagnosed patients. $J$ Cancer Res Clin Oncol, 139, 1971-84.

Hochhaus A, O’Brien SG, Guilhot F, et al (2009). Six-year follow-up of patients receiving imatinib for the first-line treatment of chronic myeloid leukemia. Leukemia, 23, 1054-61.

Hughes T, Saglio G, Branford S, et al (2009). Impact of baseline BCR-ABL mutations on response to nilotinib in patients with chronic myeloid leukemia in chronic phase. J Clin Oncol, 27, 4204-10.

Hughes T, Saglio G, Martinelli G, et al (2007). Responses and Disease Progression in CML-CP Patients Treated with Nilotinib after Imatinib Failure Appear To Be Affected by the BCR-ABL Mutation Status and Types. Am Soc Hematol, 2007.

Iqbal Z, Aleem A, Iqbal M, et al (2013). Sensitive detection of pre-existing BCR-ABL kinase domain mutations in CD34+ cells of newly diagnosed chronic-phase chronic myeloid leukemia patients is associated with imatinib resistance: implications in the post-imatinib era. PLoS One, 8, e55717.

Izzo B, Gottardi EM, Errichiello S, et al (2019). Monitoring Chronic Myeloid Leukemia: How Molecular Tools May Drive Therapeutic Approaches. Front Oncol, 9, 833.

Jabbour E, Kantarjian H, Jones D, et al (2006). Frequency and clinical significance of BCR-ABL mutations in patients with chronic myeloid leukemia treated with imatinib mesylate. Leukemia, 20, 1767-73.

Jabbour E, Kantarjian H, O'Brien S, et al (2011). Predictive factors for outcome and response in patients treated with second-generation tyrosine kinase inhibitors for chronic myeloid leukemia in chronic phase after imatinib failure. Blood, 117, 1822-7.

Jabbour E, Strati P, Cabrero M, et al (2017). Impact of achievement of complete cytogenetic response on outcome in patients with myelodysplastic syndromes treated with hypomethylating agents. Am J Hematol, 92, 351-8.

Jacobs ZG, Kaimila B, Wasswa PM, Bui T (2018). A case of massive splenomegaly due to chronic myeloproliferative neoplasm. Malawi Med J, 30, 46-8.

Kang HY, Hwang JY, Kim SH, et al (2006). Comparison of allele specific oligonucleotide-polymerase chain reaction and direct sequencing for high throughput screening of ABL kinase domain mutations in chronic myeloid leukemia resistant to imatinib. Haematologica, 91, 659-62.

Kang KH, Kim SH, Choi SY, et al (2018). Compound mutations involving T315I and P-loop mutations are the major components of multiple mutations detected in tyrosine kinase inhibitor resistant chronic myeloid leukemia. Leuk Res, 76, 87-93.

Kantarjian HM, Giles FJ, Bhalla KN, et al (2011). Nilotinib is effective in patients with chronic myeloid leukemia in chronic phase after imatinib resistance or intolerance: 24-month follow-up results. Blood, 117, 1141-5.

Karimi M, Mehrabani D, Yarmohammadi H, Jahromi F (2008) $\mathrm{S}$. The prevalence of signs and symptoms of childhood leukemia and lymphoma in Fars Province, Southern Iran. Cancer Detect Prev, 32, 178-83.

Khorashad JS, Kelley TW, Szankasi P, et al (2013). BCR-ABL1 compound mutations in tyrosine kinase inhibitor-resistant CML: frequency and clonal relationships. Blood, 121, 489-98.
Laskowski RA, Swindells MB (2011). LigPlot+: multiple ligand-protein interaction diagrams for drug discovery. ACS Publications; 2011.

le Coutre P, Ottmann OG, Giles F, et al (2008). Nilotinib (formerly AMN107), a highly selective BCR-ABL tyrosine kinase inhibitor, is active in patients with imatinib-resistant or -intolerant accelerated-phase chronic myelogenous leukemia. Blood, 111, 1834-9.

Liedtke W, Battistini L, Brosnan CF, Raine CS (1994). A comparison of methods for RNA extraction from lymphocytes for RT-PCR. PCR Methods Appl, 4, 185-7.

Marchesi V (2013). Haematological cancer: Nilotinib reduces emergence of BCR-ABL mutations in CML. Nat Rev Clin Oncol, 10, 248.

Melo JV, Barnes DJ (2007). Chronic myeloid leukaemia as a model of disease evolution in human cancer. Nat Rev Cancer, 7, 441-53.

Mueller M, Branford S, Radich J, et al (2007). Response dynamics to nilotinib depend on the type of BCR-ABL mutations in patients with chronic myelogenous leukemia (CML) after imatinib failure. J Clin Oncol, 25, 7024.

O'Hare T, Shakespeare WC, Zhu X, et al (2009). AP24534, a pan-BCR-ABL inhibitor for chronic myeloid leukemia, potently inhibits the T315I mutant and overcomes mutationbased resistance. Cancer Cell, 16, 401-12.

Ozkan T, Hekmatshoar Y, Ertan-Bolelli T,et al (2018) Determination of the Apoptotic Effect and Molecular Docking of Benzamide Derivative XT5 in K562 Cells. Anticancer Agents Med Chem, 18, 1521-30.

Rowley JD (1973). Letter: A new consistent chromosomal abnormality in chronic myelogenous leukaemia identified by quinacrine fluorescence and Giemsa staining. Nature, 243, 290-3.

Slupianek A, Poplawski T, Jozwiakowski SK, et al (2011). BCR/ $\mathrm{ABL}$ stimulates WRN to promote survival and genomic instability. Cancer Res, 71, 842-51.

Soverini S, Bavaro L, De Benedittis C, et al (2020). Prospective assessment of NGS-detectable mutations in CML patients with nonoptimal response: the NEXT-in-CML study. Blood, $135,534-41$.

Soverini S, Gnani A, Colarossi S, et al (2009). Philadelphiapositive patients who already harbor imatinib-resistant Bcr-Abl kinase domain mutations have a higher likelihood of developing additional mutations associated with resistance to second- or third-line tyrosine kinase inhibitors. Blood, 114, 2168-71.

Soverini S, Hochhaus A, Nicolini FE, et al (2011). BCR-ABL kinase domain mutation analysis in chronic myeloid leukemia patients treated with tyrosine kinase inhibitors: recommendations from an expert panel on behalf of European LeukemiaNet. Blood, 118, 1208-15.

Soverini S, Martinelli G, Colarossi S, et al (2006). Presence or the emergence of a F317L BCR-ABL mutation may be associated with resistance to dasatinib in Philadelphia chromosome-positive leukemia. J Clin Oncol, 24, e51-2.

Soverini S, Martinelli G, Colarossi S, et al (2007). Second-line treatment with dasatinib in patients resistant to imatinib can select novel inhibitor-specific BCR-ABL mutants in $\mathrm{Ph}+$ ALL. Lancet Oncol, 8, 273-4.

Ursan ID, Jiang R, Pickard EM,et al (2015) Emergence of BCR-ABL kinase domain mutations associated with newly diagnosed chronic myeloid leukemia: a meta-analysis of clinical trials of tyrosine kinase inhibitors. J Manage Care Specialty Pharm, 21, 114-22.

van Dongen JJM, Macintyre EA, Gabert JA, et al (1999). Standardized RT-PCR analysis of fusion gene transcripts from chromosome aberrations in acute leukemia for 
detection of minimal residual disease. Leukemia, 13, 1901.

Weisberg E, Manley P, Mestan J, et al (2006). AMN107 (nilotinib): a novel and selective inhibitor of BCR-ABL. Br J Cancer, 9, 1765-9.

Weisberg E, Manley PW, Cowan-Jacob SW, Hochhaus A, Griffin JD (2007). Second generation inhibitors of BCR-ABL for the treatment of imatinib-resistant chronic myeloid leukaemia. Nat Rev Cancer, 7, 345-56.

Willis SG, Lange T, Demehri S, et al (2005). High-sensitivity detection of BCR-ABL kinase domain mutations in imatinibnaive patients: correlation with clonal cytogenetic evolution but not response to therapy. Blood, 106, 2128-37.

Wu X, Kabalane H, Kahli M, et al (2018). Developmental and cancer-associated plasticity of DNA replication preferentially targets GC-poor, lowly expressed and late-replicating regions. Nucleic Acids Res, 46, 10157-72.

Yang XC, Qiu HX, Zhang SJ, et al (2014). [Treatment of two chronic myeloid leukemia patients with V299L mutation by using nilotinib]. Zhongguo shi yan xие yе хие za zhi, 22, 698-702.

\section{c) (i) (8)}

This work is licensed under a Creative Commons AttributionNon Commercial 4.0 International License.
Zhang Y (2008). I-TASSER server for protein 3D structure prediction. BMC Bioinformatics, 9, 40. 